\title{
Sample articles from Le Maître Phonétique
}

The following two texts were published by the predecessor of our journal over half a century ago and illustrate the characteristic nature of its publications at that time.

The journal, which first appeared in 1886 under the title The Phonetic Teacher /Fonètik Tîtcer/, was renamed Le Maître Phonétique /lo meitro fonetik/ in 1889. In 1971, after a lengthy debate with its readers, the journal was renamed Journal of the International Phonetic Association. Le Maître Phonétique was edited by Paul Passy until 1927, when he was succeeded by Daniel Jones. It originally appeared in four issues a year, reduced to two a year from 1942.

The official language of the journal was French, so the list of contents was given in that language, although by the time of our two samples, a large number of the papers, like these two, were in English. With a few exceptions, the contributions were written in a phonetic transcription of the author's choosing - often to reflect his or her linguistic background. It is to be noted that, unlike modern practice, the phonetic transcription is accompanied by normal punctuation, and also that all proper names are transcribed orthographically.

The journal published general articles (articles de fond /artiklə də f ̃̃/), reviews (comptes rendus $/ \mathrm{k}$ trã $\mathrm{dy} /$ ) and specimens of phonetic transcription of different languages (spécimens /spesimen/), as well as material for students (partie des élèves /parti dez ele:v/) and administrative material (partie administrative /parti administrativ/).

Our first text, entitled 'On the classification of stop consonants', is from the 1939 JanuaryMarch issue (vol. 65) and is an example of the article de fond type of contribution. It was written by J. C. Catford. ${ }^{1}$

The second text, entitled 'Dutch', is from the 1948 January-June issue (vol. 89), and illustrates the spécimen type of contribution. It was written by Eli Fischer-Jørgensen ${ }^{2}$ and J. G. Talma-Schilthuis. ${ }^{3}$ The description is brief compared to current practice, with, for example, no list of words illustrating the sounds. Unlike many of the spécimen contributions which (still today) traditionally illustrate their description with a reading of Aesop's fable 'The North Wind and the Sun', this contribution gives a transcription of a recording of colloquial speech, a telephone conversation between two Dutch women. In the transcription, unlike in the texts of the articles, proper names are also transcribed phonetically, preceded by the symbol $/ * /$.

\footnotetext{
${ }^{1}$ John Cunnison Catford (known as 'Ian'; 1917-2009), studied phonetics with Daniel Jones, Pierre Fouché and Marguerite Durand. He founded the School of Applied Linguistics in Edinburgh in the early 1950s. In 1964 he was invited to the University of Michigan, where he headed the English Language Institute and the Laboratory of Communication Studies until his retirement in 1985.

${ }^{2}$ Eli Fischer-Jørgensen (1911-2010) was a member of the Linguistic Circle of Copenhagen from 1933. She subsequently studied phonetics and became Denmark's first Professor of Phonetics at the University of Copenhagen.

${ }^{3}$ Johanna Geertruida Talma-Schilthuis (1901-1984) studied with Daniel Jones in 1921.
} 
lə

\title{
me:tro fonetik
}

\section{organ də 1 asosjasjõ fonetik ع̃ternasjonal}

\author{
sẼkãtkatriem ane də 1 asosjasjõ
}

trwazjєm seri.-dissєtjєm ane, zãvje-mars, 1939

some:r

artiklə də fõ.-on ðə klasifikeifn əv stop konsənənts (J. C. Catford .- - nout an *dervis (L. Sprague de Camp).- frðə not an welf $l l$ ænd arslændrk $h l$ (H. A. Rositzke).

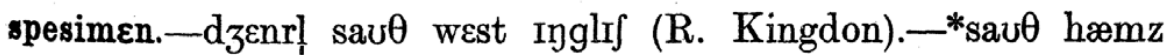
darelkkt (R. Kingdon).--kəneIdiən frentf (L. Sprague de Camp).

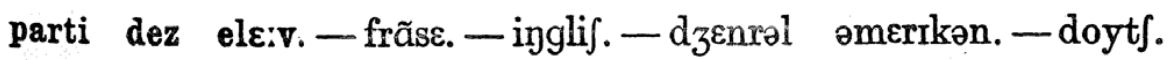
-italja:no.-espajol.

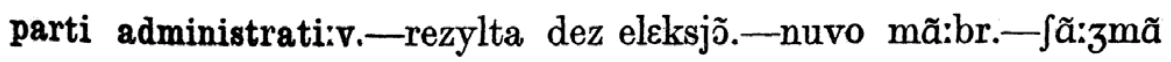
d adres. 


\section{on $\partial ә$ klasifikeifn $ә$ v stop konsənənts}

it iz wel noun ðət fənetik tə:minolədgi iz in ə ra:ðə keiətik steit. in pətikjələ, ठər iz ə lak əv sistim in ठə ju:zəl klasifikeifn ənd neimin əv stop konsənənts. fər igza:mpl, moust raitəz tend tu ki:p wət ә ju:zəli kə:ld implousivz, i:dektivz ən kliks əpa:t, әz ðou ðei dount entər intu ə dुenərəl klasifikeifn ski:m əlon wið $\Delta \partial_{\partial}$ stop konsənənts. Øər iz nou veri gud ri:zn wai ðis $\int u d$ bi:, ənd ai bili:v ðət ðə praktis iz pedəgədjikəli $\Lambda$ nsaund, in ðət it əbskjuəz ðə tru: rəleifnfips witf igzist bitwi:n o:l stop-taip kənsənənts, ənd li:dz tu veignis in stju:dnts maindz riga:din ठә mekənizm әv ठi:z saundz.

dəktə BEACH, in iz admərəbl wə:k on ðə fənetiks əv hotntot,

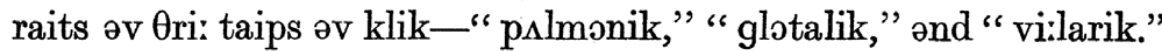
ai si: nou rizn wai ðis kaind əv klasifikeifn fudnt bi: ikstendid tu fə:m ə beisis fə diskraibiy o:l kaindz əv stəp konsənənts (ənd i:vn frikətivz). it simz tu mi: ðət s $\Delta m \theta i$ in wəd bi $d \wedge n$ to:dz klarifaiin

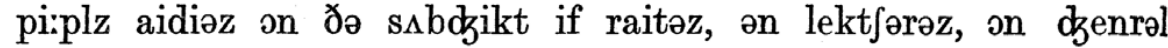

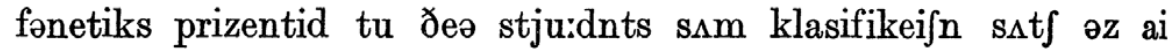
autlain hia.

ai fəd difain ə stop konsənənt $ә z$ ə spi:t-saund involvin in its prədıkfn ðə kəmplitt inklouzər əv ə bədi əv еə.

ðis bodi əv eə mei bi : (a) kəmprest $0:(b)$ reərifaid. stəp konsənənts wit involv eə kəmprefn mei bi ko:ld prefə stops, ðouz involvin reərifak $\int \mathrm{n}, \mathrm{s} \Lambda \mathrm{k} \int \mathrm{n}$ stops.

ði eə wit iz kəmprest 0 : reərifaid in prədjussin ə stop iz baundid bai ən autər ənd ən inə klouzə. ठi autə klouzər iz o:lwiz in ðə sju:preglotl ri:dgn (iksept, əf ko:s, in ठə keis əv ?, wen it iz

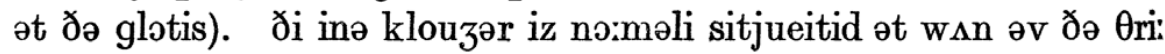

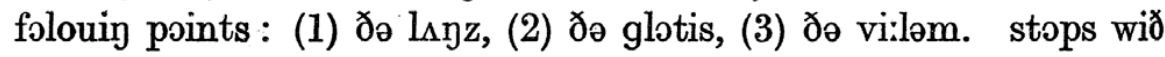
inə klouzər ət ði:z points mei bi kə:ld, әz BEACH sədjests, (1) pslmonik, (2) glotalik, ond (3) vilarik.

o:l stop kənsənənts, ðen, mei bi diskraibd bai refrens tu ði:z tu: karəktəristiks : eə kəmprefn ə: reərifakfn, ənd sitjueifn əv ði in॰ 
3

klouzə point, ənd ðeər intərəleifnfips mei bi kliəli foun in tabjulə fo:m $ð_{\Lambda} \mathrm{s}$ :

\begin{tabular}{|c|c|c|}
\hline inə klou $3^{\ominus}$ point & kəmpre $\int \mathbf{n}$ & reərifakfn \\
\hline $\ln \mathrm{z} z$ & pגlmonik prefə stops & pslmonik s $\Delta \mathrm{k} \int \mathrm{n}$ stops \\
\hline glotis & glotalik prefə stops & glotalik $\mathrm{s} \Lambda \mathrm{k} \int \mathrm{n}$ stops \\
\hline vi:ləm & vi:larik prefə stops & vi:larik s $\mathrm{k}$ kn stops \\
\hline
\end{tabular}

palmonik prefə stops (p.p. stops) a:r, əf kə:s, ði o:dinəri plousivz, p, t, k, ?, itsetra, o: voist, b, d, g, itsetrə.

palmonik sakfn stops (p.s. stops) a: simpli invə:s plousivz, prədju:st wið ikspanfn əv ठә $\theta$ orasik keidz ənd konsikwənt reərifakfn əv ði impriznd eə, rizsltin in s $\Lambda \mathrm{m}$ so:t əv implouzn ${ }^{1}$ on ðə rili:s əv ði autə klouzə.

p.s. stops a: wot wəd bi kə:ld "pslmonik kliks" bai ðouz hu: ju:z ठə tə:m " klik" tu dinout eni kaind əv sukfn stop. voist p.s. stops a: difikəlt tu prədju:s, ənd o:lmoust sə:tnli dount igzist in eni langwid 3 .

glotalik prefə stops (g.p. stops) hav ən autə klouzər in

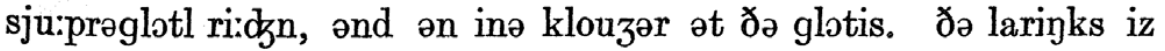
eliveitid, sou ðət $\partial_{i}$ inklouzd bodi əv eər iz kəmprest, ənd $s \Delta m$ kaind əv eksplouzh ${ }^{1}$ əkə:z wen ði autə klouzər iz rəli:st. ði:z ði ə:dinəri “ "i:dgektivz ", p', t', k', itsetrə.

voist g.p. stops du: not əkə:.

glotalik s $\Delta \mathrm{k} \int \mathrm{n}$ stops (g.s. stəps). az in $\chi_{\partial}$ keis əv g.p. stops,

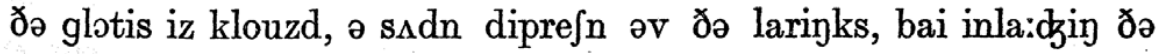
sju:prəglotl kavitiz, reərifaiz ði impriznd eə, sou ठət ən implouzn əkə:z wen $\partial \mathrm{i}$ autə klouzər iz rili:st. voislis g.s. stəps a: wot BEACH ko:lz "glotalik kliks"; ðei mait o:lsou bi ko:ld "invə:s i:dzektivz".

1 ai ju:z ठə tə:mz ' implou $3 n$ ' ənd ' eksplou $3^{n}$ ' wið ðə rekəgnaizd inglif mi:ninz

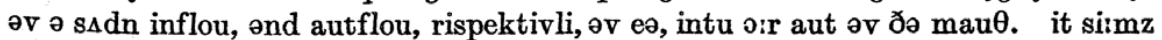

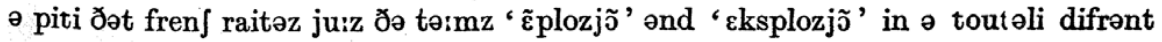

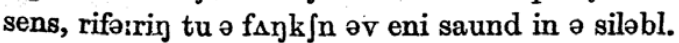


it mait bi a:gju:d ðət voist g.s. stops kanot igzist, sins voisin implaiz inkəmpli:t, э: ra:ðər intəmitnt, glotl klouzə. wət ə ju:zəli ko:ld "implousivz" kan, hauevə, bi: lidgitəmitli ko:ld "voist glotalik s $\Lambda$ kfn stops". in ðis keis ði inə klouzər iz fo:md, not bai бә klouzd glotis, bst bai бә vaibreitin voukl ko:dz. in бә prəd $\Lambda k \int n$ əv voist g.s. stops (6, d, itsetrə) ठər iz, natfərəli, ən autə klouzə in ठә sju:prəglotl eəriə. ət ठə seim taim ठə glotis iz lu:sli klouzd (fə "vois"). ðə larinks iz rapidli diprest. ə smə:l kwontiti əv еə pa:siz $\theta$ ru: ठә glotis frəm bilou, intu ठә sju:prəglotl kavitiz. ðis kwontiti əv eər iz səfifnt tu əneibl ठә voukl ko:dz tu vaibreit (prədjuisin "vois"), bst iz insəfifnt tu reiz $\chi_{i}$ eə prefər in ${ }_{i} i$ ikspandin sju:preglotl kavitiz tu $\chi_{i}$ atməsferik levl, sou ðət wen ði autə klouzər iz rilist ðеәx iz ən implouzn.

ðә mekənizm әv voist g.s. stops iz klousli ənaləgəs tu ðә fo:lt noun tu endziniəz $ә z$ " waiə dro:in ", in witf sti:m, entərin ठә siləndər əv ən enrgin wə:kin ət hai spi:d, iz "straygld", ənd $\mathrm{d} \Lambda \mathrm{z}$ nət kim in kwikli in $\Lambda$ tu ki:p $\Lambda \mathrm{p}$ шіठ бә fa:st risi:din pistən, wið ə kənsikwənt drop in prefər in бə siləndər, ənd los əv ififnsi.

vi:larik presə stops (v.p. stops) hav ən autə klouzər in ठə frsnt pa:t əv

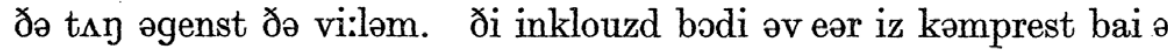

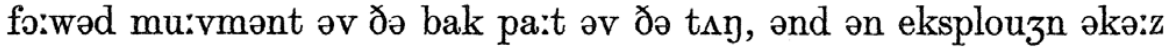
on ठә rəli:s əv ði autə klouzə. v.p. stəps ə sımtaimz kə:ld "invə:s (э:r invə:tid) kliks " bai ðouz hu: ju:z ðә tə:m "klik" in ðә sens əv " vi:larik $\mathrm{s} \Lambda \mathrm{k} \int \mathrm{n}$ stop ".

vi:larik s $\Delta \mathrm{k} \int \mathrm{n}$ stops (v.s. stops) a: fo:md wið autə klouzər in

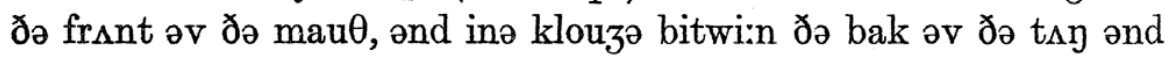
ðә vi:ləm. ði inklouzd eər iz reərifaid bai ə bakwəd mu:vmənt əv ðə bak pa:t әv ठә $\mathrm{t} \Lambda \mathrm{y}$, witf iz foloud bai ən implouzh on riliss əv ði autə klouzə.

v.s. stops a: wot ə moust komənli ko:ld “kliks" (q, c, b, itsetrə).

vi:larik stops kən nət bi vəist, in ठә ju:zəl sens əv ठə tə:m,

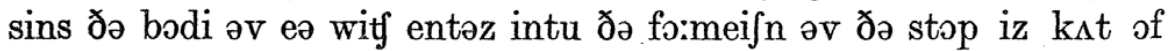
frəm ठә larinks bai ði: inə (vi:lə) klouzə. ðеi kan, hauevə, bi: 
əkımpənid bai vois. s $\Lambda$ f saundz a: ko:ld "voist kliks" bai $s \Lambda \mathrm{m}$ pi:pl, ənd kənsist əv v.s. stops pəfə:md əgenst $ə$ bakgraund $ә \mathrm{v}$ vois, in ठə fo:m әv ə vəraiəti әv g ə: y (" neizl voist kliks ").

wen glotalik prefə stops, voislis glotalik s $\Delta \mathrm{k} \int \mathrm{n}$ stops, ənd ठә vi:larik stops əkə:r in ठə tfein əv spi:t (foloud, fr instəns, bai ə vauəl saund) ठei inevitəbli involv tu: rilissiz. in ठә keis əv g.p. stops (i:djektivz), ðеәr iz ә praiməri rili:s (əv ði autə klouzə) prədjuisin $\mathrm{s} \Lambda \mathrm{m}$ kaind əv iksplouzn in ठә $\operatorname{mau} \theta$, ənd ə sekəndəri rili:s, wið ə mə:r ə: les stron glotl iksplouzn. villarik prefə stops ə:lsou hav tu: iksplousiv rili:siz, ठә sekəndəri bi:in əv ə villə taip.

voislis glotalik ənd o:l villarik $\mathrm{s} \Delta \mathrm{k} \int \mathrm{n}$ stops hav $ə$ praiməri implousiv riliss, ənd ə sekəndəri eksplousiv rili:s. ठә sekəndəri

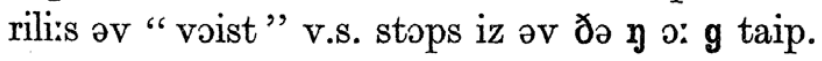

voist glotalik s $\Delta \mathrm{k} \int \mathrm{n}$ stops (implousivz), ouin tu ठә neitfər əv ðеər inə klouzə, witf iz fə:md bai ठə vaibreitin voukl ko:dz, mei bi fəloud bai vauəlz wið nou sekəndəri rili:s əv ði inə klouzə.

if dizaiəd, it iz parfiktli posibl tu ikstend ðis kaind $ә \mathrm{~V}$ klasifikeifn tu $\Lambda$ ðə saundz əz wel əz stops.

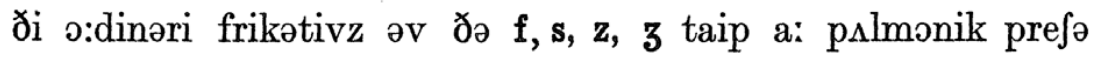
frikətivz, ənd ठә saund prədju:st wið ә $\mathrm{s} \Lambda \mathrm{dn}$ inteik əv bre $\theta, \Lambda$ ndə ði: influəns $ә \mathrm{v}$ pein, iz $ә$

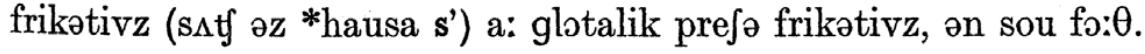

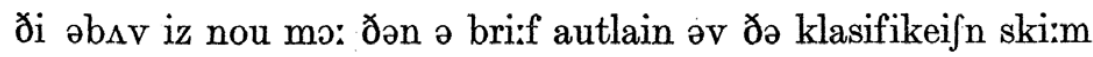

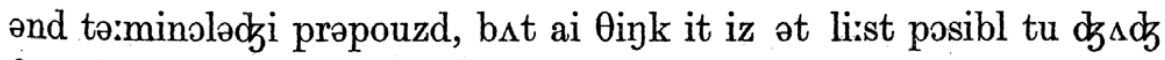
frəm it ðә kənvi:njəns əv $\mathrm{s} \Lambda \mathrm{m}$ kənsistənt sistim $\mathrm{s} \Lambda \mathrm{f}$ əz ðis.

J. C. Catford.

\section{$\theta$ nowt an *dervis}

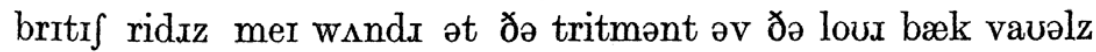
rikordid in E. B. Davis's let. in бr aktoubs-disembi, 1937, m.f., hwerin hiz prənınsieIfnz əv not, adoption, all, what I givn әz not,

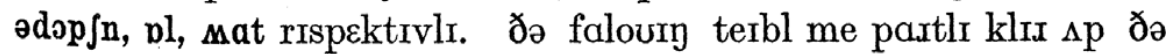
kwest $\int \mathrm{n}$ əv əmerəkən præktrs In $\mathrm{s} \Delta \mathrm{t}$ wadz : 


\title{
lə \\ meitro fonetik
}

\section{organ de 1 asosjasjõ fonetik Ẽternasjonal}

\author{
swasãttrwazjєm ane də 1 asosjasjõ
}

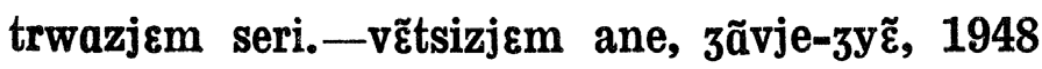

some:r

artiklə də fõ.—ðə voist viılə frikətiv æz ən inifl in, mændərin (Y. R.

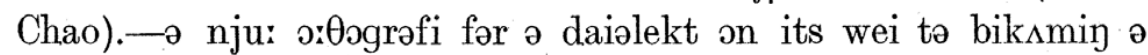

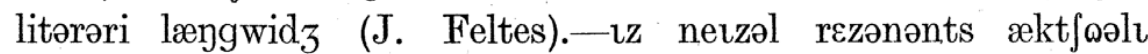
netzoœ-fərıygəl rezənənts (C. M. Wise).

spesimen. - $\operatorname{d} \Lambda \mathrm{t} \int$ (E. Fischer-Jørgensen ənd J. G. Talma-Schilthuis). kõtrãdy.-H. N. Coustenoble, La Phonétique du Provençal Moderne(J. P. Vinay). E. L. Tibbitts, A Phonetic Reader for Foreign Learners of English (D. J.).

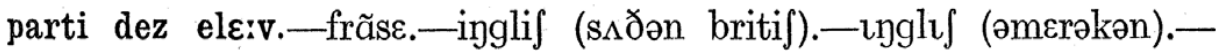
doyt .--italíano.--kasteKano.

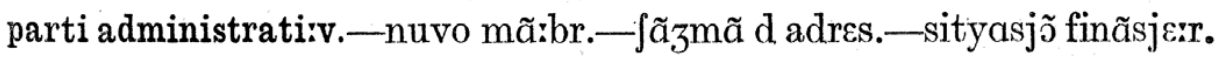


5

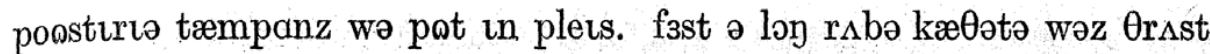

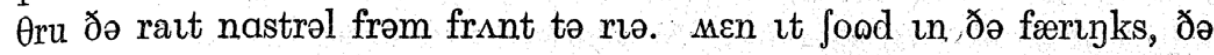

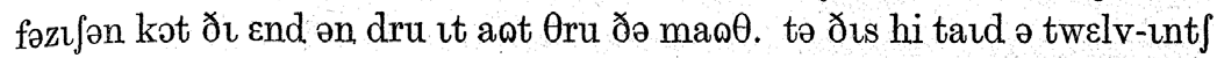

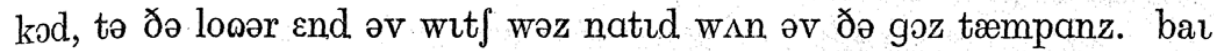

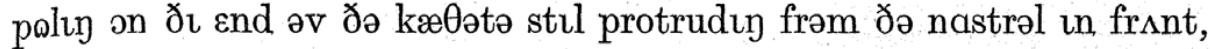

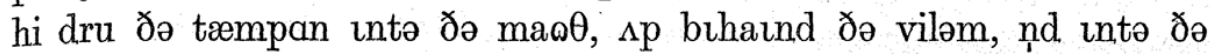

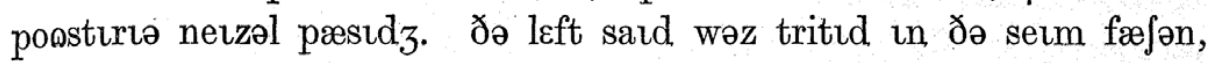

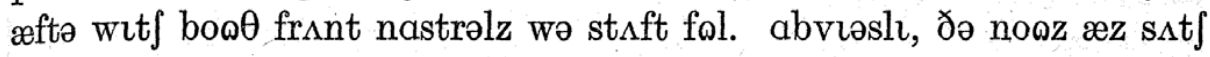

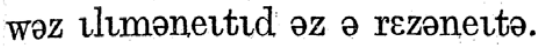

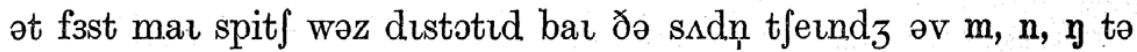

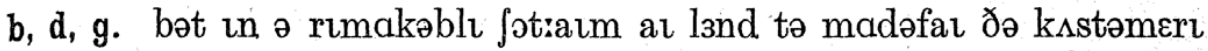

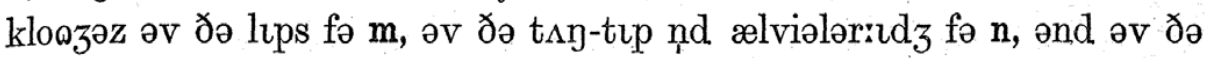

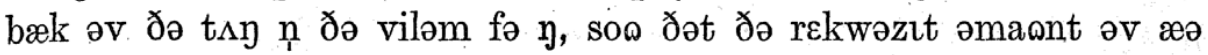

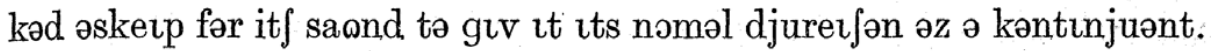
æftər ə litl præktıs, au kəd spik son nætfərəlı wıð:iz nju əd3 3 stmənts

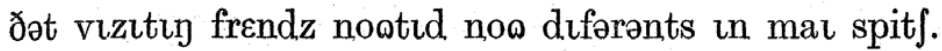

(istən əmerıkən darəlekt)

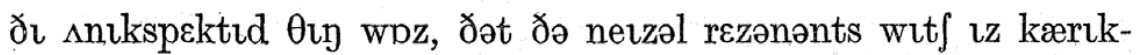

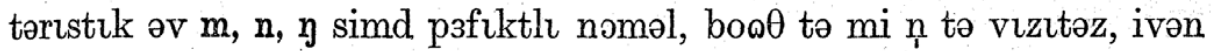

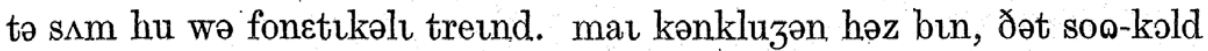

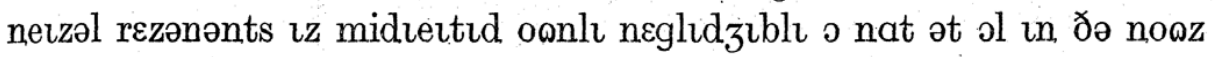
pæsıdzız propə. insted it $\mathrm{uz} \mathrm{ladzlı,} \mathrm{if} \mathrm{not} \mathrm{kəmplitl,} \mathrm{dzenəre \imath tıd} \mathrm{in}$

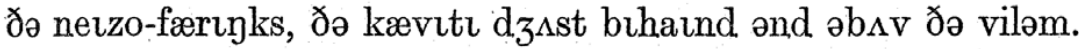

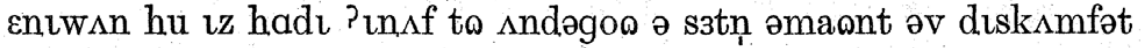

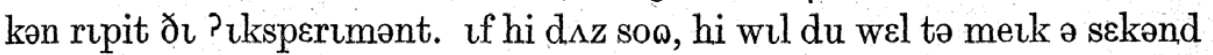

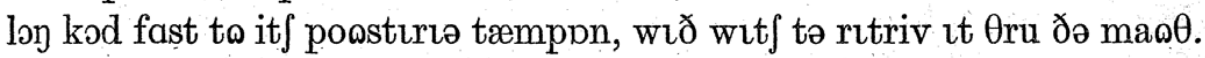

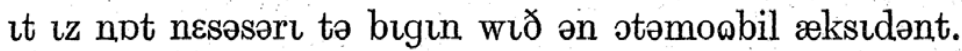

C. M. Wise.

\section{spesimen}

duts

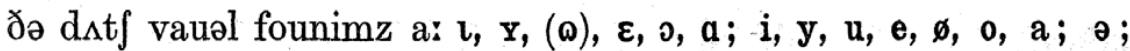
$\varepsilon i$, œi, ou.

ठә vauəlz әv ठә fərst gruip әkə:r in klouzd siləblz ounli, ðеi ə

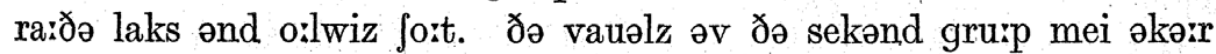
in oupn o:r in klouzd siləblz, ðеi ə ra:ðə tens ənd mei bi ley $\theta$ nd. bifo: 
r 万ei ər o:l lon, in $\Lambda$ ðə keisiz e, ø, 0, a a: ju:zəli ha:f lon, i, y, u ju:zəli fort, bət ði aktjuəl ley $\theta$ iz ra:ðə veəriəbl ənd dipendz on ठə folouin konsənənts and on stres. it iz not indikeitid in $\partial_{\partial}$ folouin tekst.

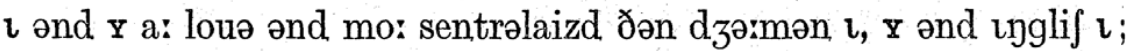
$\varepsilon$ iz ə litl əb $\Lambda \mathrm{v}$ ka:dinl. o veəriz in hait. s $\Lambda \mathrm{m}$ pirpl (bət not ठә spirkər әv ठә preznt tekst) hav tu: distinkt founimz ( w $\Lambda$ n bilou ka:dinl 0 .

i, y, u, e, $\varnothing, 0$, a a:r o:lmoust laik бә korəspondin fren $\int$ saundz. bifo: $\mathrm{r}$ e, $\varnothing$ ənd 0 a: s $\Delta$ mwot rilakst ənd saund ə litl louəd ənd sentrəlaizd.

ə iz ounli faund in $\Lambda$ nəksentid siləblz. it iz o:fn raundid ənd $\mathrm{k} \Lambda \mathrm{m} z$ ðen veri niə tu $\mathbf{x}$. it iz pəhaps not ə seprit founi:m.

$\varepsilon i$, œi, ou a: dzenrəli riga:did əz monəfounimatik (if not, œ must

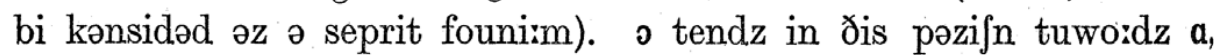
ənd $\varepsilon$ iz o:fn s $\Lambda$ mwot klousə ठən elsweə. $\propto \mathrm{d}, \Lambda \mathrm{z}$ not əkə:r əloun. it iz niə tu fren $\int \propto$, bət s $\Lambda$ mwot mo: laks ənd mo: sentrəlaizd. ठә sekənd elimənt әv ठә difӨoy œi iz dzenrəli y bifo: konsənənts, i bifo: vauəlz ənd fainəli. bifo: vauəlz ə $\mathrm{j}$ iz o:fn insə:tid a:ftə $\varepsilon \mathrm{i}$, œi, ənd ə $v$ a:ftə ou.

$\mathrm{d} \Lambda \mathrm{t} \int$ pəzesiz fərðə Әə kombineifnz aj, oj, uj (bifo: konsənənts ənd sumtaimz fainəli prənaunst ai, oi, ui), ənd iv, ev, yv (bifo: konsənənts ənd s $\Lambda$ mtaimz fainəli prənaunst iu, eu, yu).

in ə fju: forin wə:dz ðеә mei əkə: Ori: mo: vauəlz: o:, œ:, $\varepsilon$ :.

ठә dutf konsənənt founirmz a: p, t, k, b, d; f, s, x, v, z, y; v, j; $\mathrm{m}, \mathrm{n}, \mathrm{\eta} ; \mathrm{l}, \mathrm{r} ; \mathrm{h}$.

$\mathrm{p}, \mathrm{t}, \mathrm{k}$ ər nnaspireitid, b, d voist (inifəli ounli partli voist). s, z

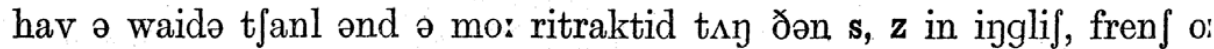
dzərmən. $\mathbf{x}, \mathbf{y}$ ər o:fn artikjuleitid veri fa: bak ənd mei bi sımwot skreipi. $\mathbf{v}$ ənd $\mathbf{z}$ (ispefəli $\mathbf{v}$ ) hav inifəli veri litl vois, ənd $\mathrm{z}$ iz o:fn kəmplistli $\Lambda$ nvoist, it mei irvn bi partli o: fuli $\Lambda$ nvoist in intəvoukalik pəzijn.

fainəli biforr a po:z o:l plousivz and frikətivz ər $\Lambda$ nvoist. if tu: plousivz o: frikətivz $\mathrm{k} \Lambda \mathrm{m}$ təgeðər in kənektid spi:t, Øər iz o:lwiz ən əsimileifn əv vois and/o: fors. if ðə seknd elimənt iz b o: $d$, ði əsimileifn iz ju:zəli rigresiv (iksept wið $\mathrm{s} \Lambda \mathrm{m}$ spikkəz in ठə keis əv prounaunz wið inifl d); in $\Lambda$ ə keisiz prougresiv. $v, j$, neizlz, likwidz ənd vauəlz mei s $\Lambda$ mtaimz meik ə fainl konsənənt əv ə prisi:din wə:d voist, ispefəli if

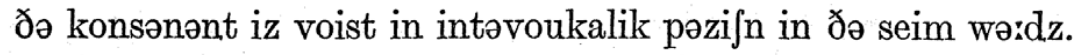

$v$ iz a veri fo:t, frikfnlis glaid. it iz dzenrəli leibioudentl, bət mei bi: baileibiəl, ənd ðеə mei bi: ə veri jo:t klouzə. 
$\mathbf{n}$ iz ə ra:ðə da:k vəraiəti a:ftə bak vauəlz. in kəloukwiəl spist $\mathbf{n}$ iz әsimileitid tə ठә folouin konsənənt əz riga:dz ठә pleis әv a:tikjuleifn. in 万i endiy -ən $\mathrm{n}$ iz ju:zəli left aut in kəloukwiəl westən $\mathrm{d} \Lambda \mathrm{t} \int \mathrm{iksept}$

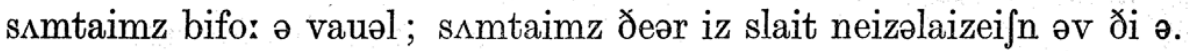

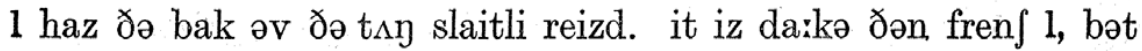
not sou da:k əz inglif da:k l. $\mathrm{r}$ iz rould, lingwəl o: ju:vjulə.

t, $\mathbf{d}$ mei bi: poustdentl o: alvioulə.

bisaidz ðizz konsənənt founirmz d $\Lambda$ t haz $\Lambda$ ðə konsənənt saundz Oru: əsimileifn: $\mathbf{g}$ ( $\mathrm{k}$ bifo: $\mathrm{b}, \mathrm{d}), \mathrm{m}$ (n o: $\mathrm{m}$ bifo: leibioudentlz), $\mathrm{p}$ ( $\mathbf{n}$ bifo: palətlz $), \int(\mathbf{s}+\mathbf{j}), \mathbf{z}(\mathbf{z}+\mathbf{j}), \mathbf{c}(\mathrm{t}+\mathbf{j})$. $\int$ ənd $\mathbf{z}$ əkə:r o:lsou in forin wə:dz.

ðә frikkwənt əkırəns əv əsimileifnz bitwinn səksesiv wə:dz iz

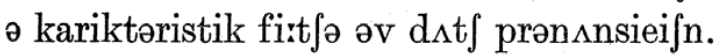

ðә mein stres iz dzenreli on ðә fərst siləbl, iksept in ठә keis әv priafiksiz kənteinin $ә$, bət ə kənsidərəbl n^mbər əv kompaund. adziktivz, hav ðə mein stres on ठә seknd elimənt. in ठә folouin tekst ði ounli igza:mplz a: helə'mal, ont'setənt ənd, œyt'stekənt.

ðə folouin tekst iz in kəloukwiəl spitt. əsimileifnz arr indikeitid

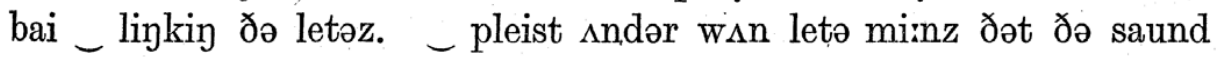
həz əridzineitid frəm ən əsimileifn.

teləfon yəsprek tysə tve nedərlansə vrouvə.

məvrou te. belt op. - y sprekt met məvrou *talma. zouvık məvrəu *molənar evə an də teləfon kynə kreijə ? - vult y evə vaxtə, məvrəu, dan zal lk məvrou molənar_upə. - hel yrax. — halo, *anəkə,

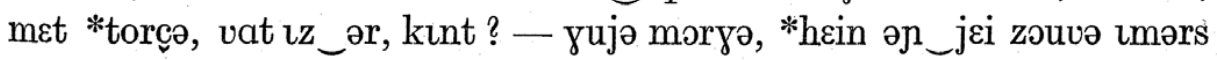
vunzdax komə lozerə. ny vuld, $\mathrm{lk}$ ¿xrax vetə hu lacyli komt, en of jə vam_plan bent fitsə me tə breyə. vantan dun və its løks. — zekər breyə və fitsə me. və stan om zevən yr vor jə nøs ən ombeitə jəzcləx

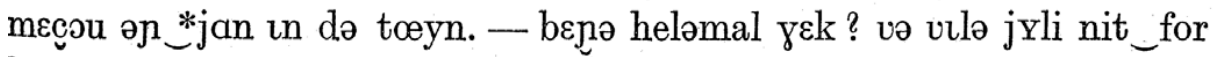
haləf nejə zin, mar dan stat ok aləs festələk klar ən vordə jyli met opə armə əntfayə. - afxəsprokə. $\iota k$ brey brodbənə əm_botər \&n ən zakjə sœykər me. - vıl ık nit hebə, mar lœystər evə verdər. ık vou botramə menemən ən op tə fits nar *lozdrext_xan. $\varepsilon$ dar un də bot.

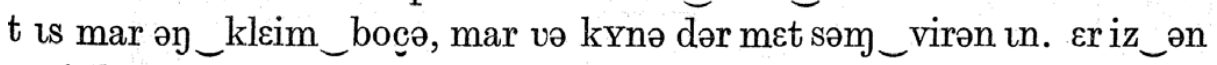

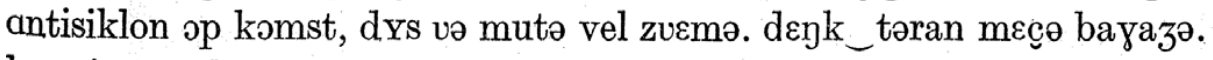
hep jə m_batmyts? - ne, mar mən har kan $\mathrm{lk}$ altzit obundə. $\mathrm{lk}$ find_ət ən enəx plan. - moi zo. və zim_vel hu lat və tœyskomə. dat hajt_fan omueron ən zo af, әm_vo etə hir. $2 k$ mak_so vel 
mogələk aləs van tə vorə klar. - - ik_fərhøx mə ontsetənt, əm_və bleivə məsxim_vel əm_vek. - destə betər. tot vunzdax an ət ombeit. jruto an *hein. - dax lifjə, yrut *jan ok_famə. tot_sins.

Elt Fischer-Jørgensen.

J. G. Talma-Schilthuis.

\section{kõtrãdy}

Hélène N. Coustenoble, La Phonétique du Provençal Moderne en Terre d'Arles (Hertford: Stephen Austin \& Sons, 1945, xii +281 pp., rəlje twal).

pøt e:trə parsə kə lə provãsal avet ete syrtu etydje $3 y$ sk isi $d \tilde{e}$

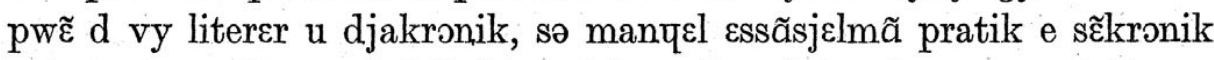

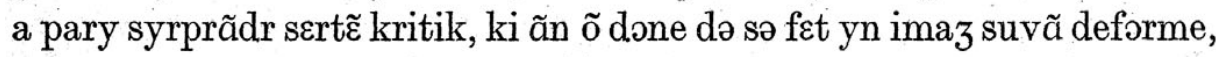
parfwa $\tilde{\varepsilon} 3 y s t$.

il a ete rprofe a 1 otœr də $\mathrm{n}$ avwar pa livre sõn uvraz o pyblik

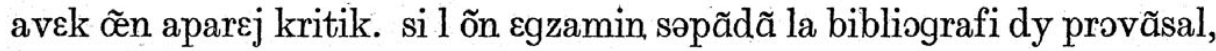
$\tilde{o}$ sə persqadra vit kə s yt ete syperfly. avã Mistral, kelkə travo d amatœr ki nə sore nuz arete; pui Mistral, ki illymin lə syze; apre

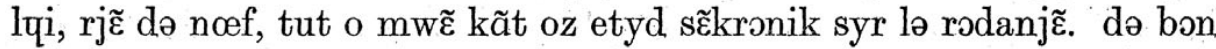

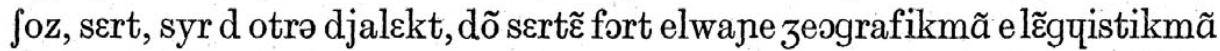
parlã də səlui kə l otœr a etydje isi : HARRY EgerTon Ford a pyblie ã 1921 œ̃n uvraz z̃terssã, Modern Provençal Phonology and Morphology, ki nə fєt okynmã dubl ãplwa avek la prezãt etyd; de monografi də Отто Zaun, Die Mundart von Aniane (Hérault), də Blinkenberg sur la patwa d'Entraumes (Alpes-Maritimes), də Georgius Sommer syr səlui də Forcalquier (Basses-Alpes) - ki dat də 1895 - e də Karl von Ettmayer syr lo djalektə də. Vinadio (Italie) . . . e la list ãn $\varepsilon$ bjẽto fet! il j oret evidamã a site de travo dy felibriz, me ki sõ ply suvãt ẽspire par de preokypasjõ literer u lokal kə par œ $\tilde{\aleph}_{\text {, susi d }}$ egzaktityd sjãtifik. lə zyzmã də D. J. mə sãblə dõk ãpləmã zystifje : sə manपel ofrə vremã đ̃ tretmã orizinal de diverz aspe fonetik d yn lãg 3yskisi bjẽ pø etydje par le filolog.

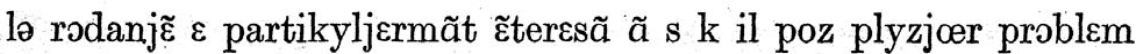
də distribysjõ fonemik. sinalõ səlui d la nazal veler $\eta$. a promjer vy, la distribysjõ de nazal $\varepsilon$ la sqivãt: $m$ e $n$ sə prezãt a l inisjal e a 1

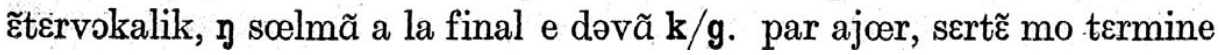

